\title{
EXTENSIONS OF FIXED POINT THEOREMS OF RHOADES AND ĆIRIĆ
}

\author{
T. K. PAL AND M. MAITI
}

\begin{abstract}
In this paper fixed point theorems have been established for the mappings which are contractive over two consecutive elements of an orbit. The results, thus established, extend the theorems of B. E. Rhoades and $\mathbf{L j}$. B. Cirić.
\end{abstract}

1. Introduction. In recent years a number of generalizations of the wellknown Banach contraction principle have appeared in the literature where the authors have introduced mappings of contractive type and studied the existence of their fixed points. A comparative study of these generalizations has been made more recently by Rhoades [1].

Wong [2] and Guseman [3] have studied the mappings which are contractive over an orbit. Here we study the mappings which are contractive only over two consecutive elements of an orbit. Fixed point theorems have been derived for such mappings as direct generalizations of the theorems established by Rhoades [1] and Ciric [4]. Examples are provided to show that the results of the present paper are indeed extensions.

2. Fixed point theorems. In the following $X$ denotes a metric space with distance function $d$. For $u \in X$, the orbit of $u$ will be denoted by $I(u, T)$, where $T$ is a self-mapping of $X$ generating the orbit. The closure of $I$ will be denoted by $\bar{I}$. The space $X$ is said to be $T$-orbitally complete if every Cauchy sequence contained in $I(u, T)$ converges in $X$, for all $u \in X$.

ThEOREM 1. Let $T$ be a mapping of a metric space $X$ into itself and $X$ be $T$-orbitally complete. If there exists an element $u \in X$ such that for any two elements $x, y \in \bar{I}(u, T)$, at least one of the following is true:

(i) $d(x, T x)+d(y, T y)<\alpha d(x, y), 1<\alpha<2$,

(ii) $d(x, T x)+d(y, T y)<\beta\{d(x, T y)+d(y, T x)+d(x, y)\}, \quad \frac{1}{2}<\beta$ $<\frac{2}{3}$,

(iii) $d(x, T x)+d(y, T y)+d(T x, T y)<\gamma\{d(x, T y)+d(y, I x)\}$, $1<\gamma<\frac{3}{2}$,

(iv) $d(T x, T y) \leqslant \delta \max \left\{d(x, y), d(x, T x), d(y, T y), \frac{1}{2}[d(x, T y)+\right.$ $d(y, T x)]\}, 0<\delta<1$.

Received by the editors August 17, 1976.

AMS (MOS) subject classifications (1970). Primary 47H10; Secondary 54H25. 
then $\left\{T^{n} u\right\}$ converges in $X$ and $\xi=\lim _{n \rightarrow \infty} T^{n} u$ is a fixed point of $T$.

Proof. Define a sequence $\left\{c_{n}\right\}$ such that $c_{n}=d\left(u_{n}, u_{n+1}\right)$, where $u_{n}=T^{n} u$ $\left(u_{0}=u\right), n=0,1,2, \ldots$ Now suppose that (i) is true for the pair $u_{n}, u_{n+1}$. Then

$$
d\left(u_{n}, u_{n+1}\right)+d\left(u_{n+1}, u_{n+2}\right)<\alpha d\left(u_{n}, u_{n+1}\right),
$$

implying

$$
c_{n+1}<(\alpha-1) c_{n} .
$$

Similarly, if (ii), (iii) and (iv) are true, then correspondingly we obtain

$$
\begin{aligned}
& c_{n+1}<[(2 \beta-1) /(1-\beta)] c_{n}, \\
& c_{n+1}<[(\gamma-1) /(2-\gamma)] c_{n}, \\
& c_{n+1}<\delta c_{n} .
\end{aligned}
$$

From (1)-(4) we observe that

$$
c_{n+1}<\lambda c_{n}
$$

for all $n$, where

$$
\lambda=\max \left\{\alpha-1, \frac{2 \beta-1}{1-\beta}, \frac{\gamma-1}{2-\gamma}, \delta\right\}<1 .
$$

It is now a simple matter to show that $c_{n} \rightarrow 0$ as $n \rightarrow \infty$ and that the sequence $\left\{u_{n}\right\}$ is Cauchy. Since $X$ is $T$-orbitally complete, then the limit $\xi$ of the sequence $\left\{u_{n}\right\}$ belongs to $X$ and also to $\bar{I}(u, T)$.

We now show that $\xi$ is a fixed point of $T$. Choose a pair of points $u_{n}$, $\xi$. Then at least one of the following holds for the pair:

(7) $d\left(u_{n}, u_{n+1}\right)+d(\xi, T \xi)<\beta\left\{d\left(u_{n}, T \xi\right)+d\left(\xi, u_{n+1}\right)+d\left(u_{n}, \xi\right)\right\}$,

(8) $d\left(u_{n}, u_{n+1}\right)+d(\xi, T \xi)+d\left(u_{n+1}, T \xi\right)<\gamma\left\{d\left(u_{n}, T \xi\right)+d\left(\xi, u_{n+1}\right)\right\}$,

$$
\begin{array}{r}
d\left(u_{n+1}, T \xi\right)<\delta \max \left\{d\left(u_{n}, \xi\right), d\left(u_{n}, u_{n+1}\right), d(\xi, T \xi),\right. \\
\left.\frac{1}{2}\left[d\left(u_{n}, T \xi\right)+d\left(\xi, u_{n+1}\right)\right]\right\} .
\end{array}
$$

As we proceed along the sequence $\left\{u_{n}\right\}$ we obtain an infinite set of values of $n$, say $\left\{n_{k}\right\}$, such that at least one of the relations (6)-(9) is satisfied by the pairs $u_{n_{k}}, \xi$. Let $k \rightarrow \infty$, we derive $d(\xi, T \xi)<0, d(\xi, T \xi)<$ $\beta d(\xi, T \xi), d(\xi, T \xi)<\gamma d(\xi, T \xi) / 2$ and $d(\xi, T \xi)<\delta d(\xi, T \xi)$ in the cases of (6), (7), (8) and (9) respectively. All these possibilities lead to the fact that $\xi$ is a fixed point of $T$. This completes the proof.

REMARK 1. If only condition (iv) is satisfied by $T$ in Theorem 1 , then $\xi$ is the unique fixed point of $T$. In other cases, however, if $T$ further satisfies at least one of the conditions

(v) $d(\xi, T x)<d(x, \xi)+d(x, T x)$, 
(vi) $d(x, \xi)<d(\xi, T x)+d(x, T x)$,for all $x(\neq \xi) \in X$, then the unicity of the fixed point is guaranteed.

The mapping $T$ satisfying (iv) has been studied first by Ciric [4] and then by Rhoades [1]. Theorem 1 indeed extends the results of Rhoades [1] and Cirić [4] and this may be seen from

EXAMPLE 1. Let $X=\{1,2,3,4,5\}$ with the distance function $d$ defined by

$$
\begin{array}{llll}
d(1,2)=4.3, & d(1,3)=0.6, & d(1,4)=3.65, & d(1,5)=2.8, \\
d(2,3)=3.95, & d(2,4)=3.7, & d(2,5)=5.0, & d(3,4)=3.9, \\
& d(3,5)=3.35, & d(4,5)=1.9 .
\end{array}
$$

Let $T$ be a mapping of $X$ into itself such that

$$
T 1=2, \quad T 2=3, \quad T 3=4, \quad T 4=5=T 5 .
$$

This example satisfies all the conditions of Theorem 1 with $\alpha=1.99$ and $\delta=0.92$. But the theorems of Rhoades and Ciric do not hold for this example since $T$ does not satisfy (iv) for $x=1, y=4$ and $x=1, y=5$.

It may be noted that the principle of quasi-contraction of Ćiric [5] also does not hold for this example since

$$
d(T 1, T 4)>\max \{d(1,4), d(1, T 1), d(4, T 4), d(1, T 4), d(4, T 1)\} .
$$

REMARK 2. Without affecting the conclusions of Theorem 1, condition (iv) may be split into its four constituent alternatives:

$$
\begin{array}{ll}
\text { (vii) } & d(T x, T y)<\rho d(x, y), \quad 0<\rho<1, \\
\text { (viii) } & d(T x, T y)<\sigma d(x, T x), \quad 0<\sigma<1, \\
\text { (ix) } & d(T x, T y)<\mu d(y, T y), \quad 0<\mu<1, \\
\text { (x) } & d(T x, T y) \leqslant \nu\{d(x, T y)+d(y, T x)\}, \quad 0<\nu<\frac{1}{2} .
\end{array}
$$

Remark 3. The existence and uniqueness of the fixed point of $T$ may be ensured if some iterate of $T$ satisfies the conditions of Theorem 1 along with (v) or (vi).

Conditions (i)-(iv) of Theorem 1, respectively, imply the following:

(b) $d(x, T x)+d(y, T y)<\frac{2}{3}\{d(x, T y)+d(y, T x)+d(x, y)\}$,

$$
\begin{gathered}
d(x, T x)+d(y, T y)+d(T x, T y)<\frac{3}{2}\{d(x, T y)+d(y, T x)\} \\
d(T x, T y)<\max \{d(x, y), d(x, T x), d(y, T y), \\
\left.\frac{1}{2}[d(x, T y)+d(y, T x)]\right\} .
\end{gathered}
$$

These lead to the following generalization of Theorem 1, which is close in spirit to Edelstein's generalization [6] of Banach's theorem.

TheOREM 2. Let $T$ be a mapping of a metric space $X$ into itself. If

(i) there exists a point $u \in X$ such that the orbit $I(u, T)$ has a cluster point $\xi \in X$, 
(ii) $T$ and $T^{2}$ are continuous at $\xi$,

(iii) $T$ satisfies (a) or (b) or (c) or (d) for any two distinct elements $x, y \in \bar{I}(u, T)$,

then $\xi$ is a fixed point of $T$.

Proof. Setting $c_{n}$ as in Theorem 1 it is possible to show that $c_{n+1}<c_{n}$ for all cases (a)-(d). Therefore the sequence $\left\{c_{n}\right\}$ is monotone decreasing and bounded also. Then $c_{n} \rightarrow l$ as $n \rightarrow \infty$, where $l=\inf \left\{c_{n}\right\}$.

Since $\xi$ is a cluster point of $\left\{u_{n}\right\}$, there exists a subsequence $\left\{u_{n_{n}}\right\}$ such that $u_{n_{i}} \rightarrow \xi$ as $i \rightarrow \infty$. Also $u_{n_{i}+1}=T u_{n_{i}} \rightarrow T \xi$ and $u_{n_{i}+2}=T^{2} u_{n_{i}} \rightarrow T^{2} \xi$ as $i \rightarrow \infty$, because $T$ and $T^{2}$ are continuous at $\xi$. Then we obtain

$$
\begin{aligned}
& l=\lim _{i \rightarrow \infty} d\left(u_{n_{i}}, u_{n_{i}+1}\right)=\lim _{i \rightarrow \infty} d\left(u_{n_{i}}, T u_{n_{i}}\right)=d(\xi, T \xi), \\
& l=\lim _{i \rightarrow \infty} d\left(u_{n_{i}+1}, u_{n_{i}+2}\right)=\lim _{i \rightarrow \infty} d\left(T u_{n_{i}}, T^{2} u_{n_{i}}\right)=d\left(T \xi, T^{2} \xi\right) .
\end{aligned}
$$

Suppose that $\xi \neq T \xi$. If (a) holds for the pair $\xi, T \xi$, then

$$
d(\xi, T \xi)+d\left(T \xi, T^{2} \xi\right)<2 d(\xi, T \xi) \text {, i.e. } d\left(T \xi, T^{2} \xi\right)<d(\xi, T \xi),
$$

which is impossible. Hence $T \xi=\xi$. If (b) or (c) or (d) holds for this pair instead of (a), then it is also possible to derive that $T \xi=\xi$. Thus $\xi$ is a fixed point of $T$. This completes the proof.

The statements corresponding to those in Remarks 1 and 3 are also true in the case of the above theorem. Finally, we may note that condition (d) may be replaced by its constituent alternatives corresponding to (vii)-(x).

ACKNOWLEDGEMENT. The authors wish to thank the referee for his useful suggestions which have greatly improved the paper.

\section{REFERENCES}

1. B. E. Rhoades, A comparison of various definitions of contractive mappings, Trans. Amer. Math. Soc. 226 (1977), 256-290.

2. J. S. W. Wong, Two extensions of the Banach contraction mapping principle, J. Math. Anal. Appl. 22 (1968), 438-443. MR 37 \#4682.

3. L. F. Guseman, Jr., Fixed point theorems for mappings with a contractive iterate at a point, Proc. Amer. Math Soc. 26 (1970), 615-618. MR 42 \#919.

4. Lj. B. Ciric, Generalized contraction and fixed point theorems, Publ. Inst. Math. (Beograd) (N.S.)12 (26) (1971), 19-26. MR 46 \#8203.

5., A generalization of Banach's contraction principle, Proc. Amer. Math. Soc. 45 (1974), 267-273. MR 50 \#8484.

6. M. Edelstein, On fixed and periodic points under contractive mappings, J. London Math. Soc. 37 (1962), 74-79. MR 24 \# A2936.

Department of Mathematics, Kharagpur College, Kharagpur, India

Departiment of Mathematics, Indian Institute of Technology, Kharagpur, India 